Abanico Veterinario. Enero-Diciembre 2020; 10(1):1-10. http://dx.doi.org/10.21929/abavet2020.3 Artículo Original. Recibido: 09/08/2019. Aceptado: 20/01/2020. Publicado: 15/02/2020.

\title{
Prevalencia de Fasciola hepatica en ganado bovino de Lidia
} Prevalence of Fasciola hepatica in Lidia bovine breed

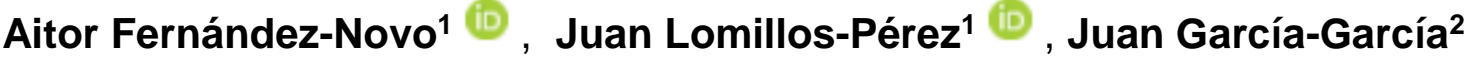

1Departamento de Producción y Sanidad Animal, Salud Pública Veterinaria y Ciencia y Tecnología de los Alimentos. Facultad de Veterinaria. Universidad Cardenal Herrera-CEU. Tirant lo Blanc, 7. CP 46115. Alfara del Patriarca - Valencia. España. 'La Tejera Sociedad Limitada Profesional. De la cruz, 15. CP 28710. El Molar - Madrid. España. Autor responsable: Fernández-Novo, Aitor. Autor de correspondencia: Fernández-Novo, Aitor. Cuerda larga 13. CP 28413. El Boalo, Madrid, España. aitor.fernandeznovo@uchceu.es, juan.lomillos@uchceu.es, veterinariogarciagarcia@gmail.com

\section{RESUMEN}

La fasciolosis sigue siendo una parasitosis frecuente dentro del bovino en extensivo, a pesar de la gran implantación y eficacia de fármacos fasciolicidas. En el presente trabajo se ha estudiado durante cinco años la prevalencia de Fasciola hepatica analizando 251 bovinos de lidia, evaluando la presencia de lesiones macroscópicas hepáticas. A su vez, su relación con la temperatura y pluviometría media del año, y describir sus consecuencias fisiopatológicas durante el festejo taurino. Metodología: se llevó a cabo en Madrid (julio-septiembre 2013-2017). Resultados: $n=251$ animales en los cinco años, $50.20 \pm 2.68$ evaluados al año, de los cuales, 14 presentaron lesiones, de media $2.80 \pm 1.64$ al año. Asimismo, se reseñaron en el reconocimiento un total de 4 animales compatibles con lesiones en los cinco años, de media $0.80 \pm 0.84$ animales al año. La temperatura media anual fue de $15.78 \pm 0.48^{\circ} \mathrm{C}$ y la pluviometría media anual de $570.20 \pm 102.21 \mathrm{~mm}$. Se observa correlación estadística entre las canales con lesiones y la pluviometría media anual $(P<0.01)$ y con la temperatura media anual $(P<0.05)$. Se obtienen prevalencias medias $(5.61 \pm 2.82)$ inferiores a las descritas por diferentes autores, junto con condiciones climatológicas compatibles con el crecimiento del parásito y de todos sus hospedadores intermediarios. El estudio revela que un buen uso de los antiparasitarios no es del todo efectivo en este tipo de explotaciones.

Palabras clave: fasciolosis, temperatura, pluvimetría, parásito, toro, lidia y bovino.

\begin{abstract}
Fasciolosis remains a relatively frequent parasitic infection in cattle, despite the great implantation and efficacy of fasciolicides. In the present work, the prevalence of Fasciola hepatica has been observed for five years, analyzing 251 bullfighting cattle, evaluating the presence of macroscopic lesions in the liver. Moreover, the relationship with the temperature and average rainfall of the year, and also it describes the pathophysiological consequences during bullfighting. Methodology: it was carried out in Madrid (July-September 2013-2017). Results: $n=251$ animals in five years, 50.20 \pm 2.68 evaluated per year, which 14 had lesions, on average $2.80 \pm 1.64$ per year. Likewise, a total of 4 compatible animals with lesions were reported during the five years, with an average of $0.80 \pm 0.84$ animals per year. The average annual temperature was $15.78 \pm 0.48^{\circ} \mathrm{C}$. The average annual rainfall was $570.20 \pm 102.21 \mathrm{~mm}$. A statistical correlation was observed between the carcasses with lesions and the average annual rainfall $(P<0.01)$ and the annual mean temperature $(P<0.05)$. The experiment revealed lower prevalence $(5.61 \pm 2.82)$ than the one described by different authors, having also compatible climatological conditions with the growth of the parasite and all its intermediate hosts. The study reveals that a good praxis using antiparasitic drugs is not entirely efficient.
\end{abstract}

Keywords: fasciolosis, temperature, pluviometry, parasite, Lidia bullfight, bovine. 


\section{INTRODUCCIÓN}

La primera referencia que se conoce de Fasciola hepatica, corresponde a una explotación de ganado ovino sita en Francia, propiedad de Jean de Brie; que describe en 1379, una enfermedad de las ovejas en las que el hígado se pudre por el consumo de ranunculáceas y cría gusanos grandes y planos. En 1774 Weinland descubre que el hospedador intermediario era el molusco Lymnae trunculata. Pero no sería hasta 1892 cuando se descubrió que los rumiantes se infectaban al ingerir larvas enquistadas en la hierba (metacercarias). En 1883 A.P. Thomas y R. Leuckart descubrieron el ciclo biológico completo, morfología y la biología del parásito. $F$. hepatica (reino: animalia, filo: platyhelminthes, clase: trematoda, subclase: digenea, orden: echinostomida, familia: fasciolidae, género: fasciola, especie: $F$. hepatica. Linnaeus, 1758) es un parásito eurixeno, tiene un amplio espectro de hospedadores definitivos; los principales son los rumiantes, pero también puede parasitar a otros vertebrados mamíferos, como: équidos, suidos, roedores, lagomorfos; incluso carnívoros y marsupiales. También primates, y por lo tanto el propio el hombre. Se trata de una zoonosis, aunque no muy prevalente en España y Europa; en otras partes del planeta es relativamente frecuente (Cordero, 1990).

Respecto al hospedador intermediario, es estenoxeno; únicamente en las zonas templadas del planeta se ubica; actúa como hospedador intermediario, un caracol acuático: Lymnaea (Galba) truncatula. L. truncatula, determina la existencia de este proceso en zonas húmedas. En las heces de los rumiantes infectados con $F$. hepatica se liberan huevos al medio, que son no embrionados; sufren un proceso de embrionación en el ambiente, cuando las condiciones de temperatura y humedad son adecuadas, en un periodo de tiempo que oscila de 2 a 4 semanas; necesitan humedad y temperaturas superiores a los $10^{\circ} \mathrm{C}$. (Cordero, 1990).

Las formas larvarias, una vez maduras, eclosionan del huevo, y se denominan miracidios. Estos nadan activamente en el agua y se dirigen al hospedador intermediario, penetran a través de la musculatura del pie en el caracol, y en el interior tienen lugar las siguientes fases del ciclo biológico, que son fases de multiplicación asexual: esporocisto, redia y cercaria. Estas fases, en condiciones óptimas tardan en desarrollarse en torno a los 40 días. Las cercarias salen del hospedador intermediario, vuelven a nadar dirigiéndose a la vegetación que se encuentra en los márgenes de zonas encharcadas, donde se enquistan; dando lugar a una forma de resistencia llamada metacercaria. Las metacercarias serán ingeridas de nuevo por el hospedador definitivo (Claridge et al., 2012).

El ciclo biológico endógeno comienza por la ingestión de las metacercarias. Por la acción de la temperatura corporal, del ambiente anaerobio del tracto digestivo y de las enzimas biliares, se produce el desenquistamiento de las fases juveniles de $F$. hepatica; y comienza la migración desde duodeno, hasta la localización definitiva, los conductos biliares (Rojo-Vázquez, 2012). La migración se produce pasando del duodeno a cavidad abdominal, y a partir de los 4-6 días post-infección llegan al hígado. 
Desde la cavidad abdominal atraviesan la cápsula de Glisson, acceden al parénquima hepático y por último llegan a los conductos biliares; llevando a cabo una migración centrípeta (Albuquerque et al., 2013). El periodo de prepatencia es de 8-9 semanas.

La principal presentación de fasciolosis en ganado bovino de lidia, es en un cuadro clínico crónico (Clery et al., 1996; Forbes et al., 2015). Además, debemos recordar que la principal aptitud del ganado de lidia se fundamenta en los festejos taurinos; por lo tanto, en este momento de ejercicio máximo, intenso y prolongado, el desempeño del animal debe ser máximo (Mulone 1986; Escribano, et al 2010; Escalera-Valente et al 2013).

El ejercicio intenso al que se somete al bovino durante el festejo, requiere una gran respuesta orgánica: aumento de capacidad pulmonar, aumento de frecuencia cardiaca y respiratoria, aumento de la disponibilidad de glucosa en torrente sanguíneo; por lo tanto, aumento de gluconeogénesis y reducción de glucogenogénesis, aumento de transformación de ácidos pirúvico, butírico y láctico; así como activación de mecanismos compensadores de acidosis metabólica por aumento de hidrogeniones (Hiney, et al 2004). En varios estudios, se demuestra la capacidad adaptativa que tiene el bovino de raza de lidia para enfrentarse a estas situaciones extremas; no obstante, determinadas circunstancias patológicas, como puede ser la fasciolosis, que mermen sus capacidades de adaptación, repercuten muy negativamente sobre su desempeño en el festejo (García-Sacristán et al., 1996; Cunningham, 2003). En primer lugar, el cuadro clínico producido en los casos agudos, puede cursar con defectos en el trapío del animal, pérdida de peso y aparición de edemas; lo cual repercutirá muy negativamente en el reconocimiento del animal previo al festejo, pues pone en compromiso su aptitud para la lidia, por no reunir las características morfométricas pertinentes (Real Decreto 60/2001, Boletín Oficial del Estado, 2001).

Las presentaciones crónicas reducirán la funcionalidad hepática de manera directamente proporcional al grado de infestación del animal; de tal forma, que una reducción en la actividad hepática repercutirá en un descenso de la síntesis de proteínas plasmáticas, produciendo generalmente hipoalbuminemia, la cual se manifiesta externamente con la presencia de edemas en zonas declives, por la extravasación de fluidos del torrente sanguíneo. Además, se reduce la síntesis y liberación de enzimas hepáticas y el valor hematocrito (Da Silva et al., 2017). Dicho valor, durante el desempeño de la lidia, es fundamental, pues en el ejercicio extenuante que realiza el animal, necesita gran cantidad de oxígeno para responder positivamente a las necesidades metabólicas (Kaneko et al., 1997).

Además, la fasciolosis lleva consigo un desequilibrio metabólico que hace aumentar el consumo de reservas de glucógeno, con una consiguiente disminución de la disponibilidad de glucógeno hepático y muscular; así como de depósitos grasos (Pérez et al., 1992). Todos ellos importantes en el fenómeno de gluconeogénesis, tan necesario para el animal durante el espectáculo taurino.

Por otro lado, lesiones crónicas de $F$. hepatica, pueden producir peritonitis, que puede ser manifiesta durante la lidia con episodios de dolor por parte del animal; así como 
con reticencia a determinados movimientos por aflicciones en cavidad abdominal (Clery et al., 1996; Forbes et al., 2015). También pueden aparecer adherencias a nivel craneal del hígado, junto con el diafragma; las cuales son inversamente proporcionales a la movilidad del diafragma, y por lo tanto, influirán negativamente en la respiración del animal y en consecuencia disminuirán la respuesta al ejercicio intenso al que se somete al bovino durante el espectáculo (Marcos et al., 2007; Lomillos et al., 2018).

En relación al tratamiento de la fasciolosis, numerosos estudios han revelado el efecto de diferentes grupos farmacológicos, que tienen efecto sobre los diferentes estadíos del parásito; los más empleados, son: nitroxinil, clorsulón, albendazol, triclabendazol y closantel (Graig y Jhuey, 1984; Mooney et al., 2009).

El objetivo del estudio fue determinar la prevalencia de Fasciola hepatica en el ganado bravo lidiado durante cinco años, mediante la presencia de lesiones macroscópicas en hígados vistos en desolladeros, su relación con la temperatura y pluviometría media del año, y sus consecuencias fisiopatológicas que merman la aptitud del animal durante el festejo taurino.

\section{MATERIAL Y MÉTODOS}

El trabajo se llevó a cabo en la comunidad de Madrid, en los festejos taurinos que tuvieron lugar durante los meses de julio, agosto y septiembre, de los años 2013 al 2017. Se analizaron un total de 251 canales, en diferentes municipios de la comunidad autónoma, todos ellos en lugares y salas autorizadas para el desollado de los animales tras el festejo (Real Decreto 260/2002, de 8 de marzo). Los animales lidiados fueron vacas de suelta (hembras entre dos y doce años de edad), novillos para capea (tres años), toros para corrida de toros (machos entre cuatro y cinco años de edad) y novillos para novilladas picadas (machos tres años) y no picadas (machos de dos años).

En el reconocimiento se señalan aquellos animales que poseen bajos niveles de condición corporal, con pelo hirsuto, sin brillo y despigmentado, signos compatibles con enfermedades crónicas, entre ellas la fasciolosis. Concluido el festejo, en el desolladero, se analizan los ganglios periportales, la superficie hepática, parénquima hepático, conductos biliares y vesícula biliar. Todos aquellos que presentaron parasitosis in situ o signología compatible con fasciolosis, como: trayectos migratorios larvarios en el hígado, de carácter hemorrágico y con necrosis coagulativa, colangiohepatitis, colengiectasia, engrosamiento y fibrosis de los conductos biliares, se consideran como hígados parasitados con $F$. hepatica, ver figura 1 . En la imagen $A$, se observan trayectos de tejido conjuntivo en parénquima hepático compatibles con migración antigua de Fasciola hepatica, y en la $\mathrm{B}$ y $\mathrm{C}$ se observan trayectos de tejido conjuntivo en superficie y parénquima hepático compatibles con migración reciente de Fasciola hepatica, así como visualización del parásito. En la imagen $B$, también se observa ligera perihepatitis fibrinosa. Se hace un recuento de la prevalencia de afectaciones hepáticas compatibles con fasciolosis en todas sus formas clínicas, frente a los que no presentan alteraciones macroscópicas compatibles con el parásito. 

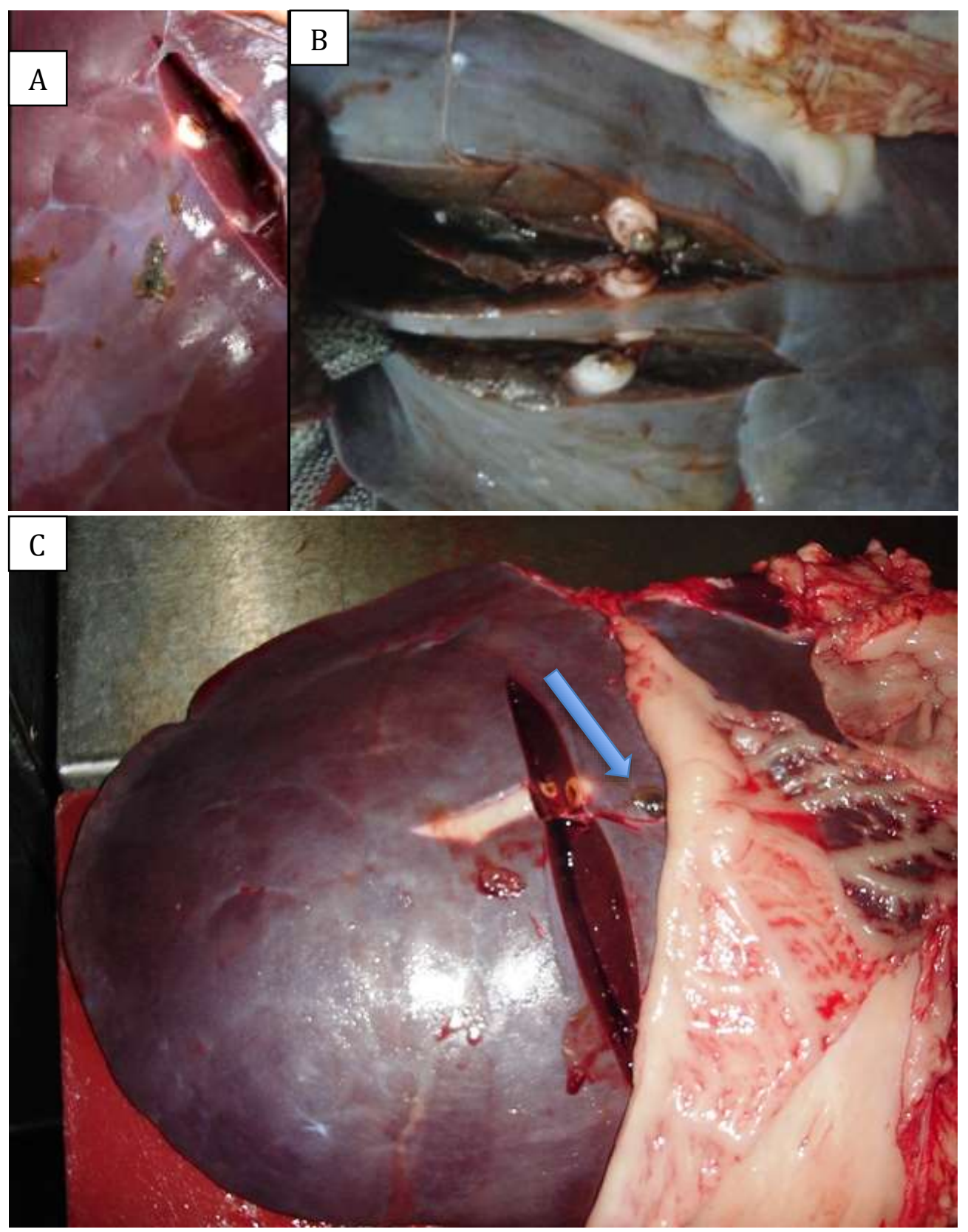

Figura 1.- Trayectos de tejido conjuntivo en parénquima hepático compatibles con migración de Fasciola hepatica, así como visualización del parásito

Se recogen todos los datos informáticamente, así como se obtienen datos de la Agencia Estatal de Meteorología (AEMET) sobre temperaturas y pluviometría medias anual. Posteriormente se realiza análisis estadístico con IBM SPSS Statistics Base 22.0. La distribución de las variables numéricas fue realizada a través del análisis tStudent; mientras que la correlación se analizó con el método de correlación de Pearson.

\section{RESULTADOS}

El número total de animales muestreados fue de 251 en los cinco años, distribuyéndose en $50.20 \pm 2.68$ evaluados al año, dentro de los cuales aparecieron lesiones en un total de 14 canales, de media $2.80 \pm 1.64$ al año. Asimismo, se 
reseñaron en el reconocimiento un total de 4 animales compatibles con lesiones en total, de media $0.80 \pm 0.84$ animales al año. La temperatura media anual fue de $15.78 \pm 0.48^{\circ} \mathrm{C}$ y la pluviometría media anual de $570.20 \pm 102.21 \mathrm{~mm}$, ver tabla 1 .

Tabla 1. Resultados obtenidos del muestreo de las canales de festejos taurinos de la zona norte de la Comunidad de Madrid durante los años 2013 al 2017

\begin{tabular}{llllllll}
\hline Año & $\mathrm{n}^{\circ}$ animales & $\begin{array}{l}\mathrm{n}^{\circ} \text { animales con } \\
\text { lesiones } \\
\text { desolladero }\end{array}$ & en & & $\begin{array}{l}\mathrm{n}^{\circ} \text { animales } \\
\text { sintomatología } \\
\text { compatible }\end{array}$ & $\begin{array}{l}\text { con } \\
\begin{array}{l}\text { Temperatura } \\
\text { media anual } \\
\left({ }^{\circ} \mathrm{C}\right)\end{array}\end{array}$ & $\begin{array}{l}\text { Pluviometría } \\
\text { media anual } \\
(\mathrm{mm})\end{array}$ \\
\hline 2013 & 50 & 2 & $4.00 \%(2 / 50)$ & 2 & 14.97 & 515 \\
2014 & 46 & 4 & $8.69 \%(4 / 46)$ & 1 & 15.96 & 680 \\
2015 & 53 & 2 & $3.77 \%(2 / 53)$ & 0 & 16.00 & 500 \\
2016 & 52 & 5 & $9.61 \%(5 / 52)$ & 1 & 15.80 & 682 \\
2017 & 50 & 1 & $2.00 \%(1 / 50)$ & 0 & 16.20 & 474 \\
Media & 50.20 & 2.80 & $5.61 \%$ & 0.80 & 15.78 & 570.20 \\
Desviación & 2.68 & 1.64 & 2.82 & 0.84 & 0.48 & 102.21 \\
estándar & & & & & & & \\
\hline
\end{tabular}

El número de animales con lesiones en desolladero compatibles hallados, no guarda relación estadísticamente significativa con el número total de animales evaluados al año $(P=0.159)$, ni con los animales que presentaron sintomatología compatible en el reconocimiento $(P=0.327)$. En cambio, sí existe significancia estadística entre el número de animales con lesiones en desolladero compatibles hallados y la pluviometría media anual $(P<0.01)$ y con la temperatura media anual $(P<0.05)$.

\section{DISCUSIÓN}

La prevalencia de fasciolosis en vacuno lechero en un estudio realizado en 275 rebaños en Galicia durante el año 2007, reveló unos datos de prevalencia de $61.1 \%$, sin encontrar diferencias estadísticamente significativas entre rebaños tratados y no tratados con diferentes fármacos (Piñeiro $P, 2013$ ).

En el presente estudio se ha obtenido una prevalencia de $5.61 \pm 2.82$ animales con lesiones compatibles al año, cifra significativamente muy inferior al estudio publicado por Orjales et al., 2017. Cabe reseñar la importancia del empleo de fármacos fasciolicidas, previos a la predominante temporada de espectáculos taurinos: junio a septiembre, en comparación con los fármacos usados en el estudio anterior que se aplicaron de manera continuada durante todo el año, en el periodo seco del vacuno lechero. No obstante, la eficacia de los tratamientos antiparasitarios es dependiente en función de la estrategia de aplicación; así como deben considerarse las posibles resistencias antiparasitarias (Kelley et al., 2016; Beesley et al., 2017). Los datos que existen sobre fasciolosis en ganado bovino extensivo, reflejan una alta prevalencia del parásito (Orjales et al., 2017; González-Lanza et al. 1989), describen en León una prevalencia del $29.5 \%$ de animales infectados, así como una relación íntima entre prevalencia y edad de los animales. Dicha relación tiene significación en ganado de lidia, pues Castilla-León presenta un elevado de porcentaje de ganaderías de vacuno de lidia; y además, un amplio porcentaje de animales se lidian con cuatro, cinco y seis años de edad. 
No obstante, también cabe destacar la diferencia climatológica de la cornisa cantábrica española, donde hay menor representación de ganaderías de vacuno de lidia, en relación a la climatología del centro y sur peninsular, donde se encuentran la amplia mayoría de explotaciones de ganado bovino (Lomillos et al., 2012; Painceira, 2012). Los datos medios obtenidos tanto de temperatura como de pluviometría fueron $15.78 \pm 0.48^{\circ} \mathrm{C}$ y $570.20 \pm 102.21 \mathrm{~mm}$ respectivamente, los cuales se encuentran dentro de los rangos y estándares compatibles con el crecimiento de las fases larvarias, juveniles y adultas de $F$. hepatica, así como de todos sus hospedadores intermediarios, pues se establece un rango de temperatura favorable: las comprendidas entre 10 y $30^{\circ} \mathrm{C}$, con una temperatura óptima de $27^{\circ} \mathrm{C}$. (Rowcliffe, et al., 1960; García-Rodríguez et al., 1985; Astiz-Blanco et al., 2007). Sin embargo, otro estudio obtiene los mejores resultados en temperatura de $25^{\circ} \mathrm{C}$ (Diez y Rojo-Vázquez, 1976), dicha temperatura se consigue, en la zona predominante de explotaciones de vacuno de lidia, en las estaciones de primavera y verano. Dichos valores están refrendados por estudios más recientes, que evalúan la carga larvaria en pastos a lo largo del año, siendo máximas en los meses de primavera y verano (Nogareda et al., 2006). Los resultados podrían estar justificados por una mayor pluviometría en esos años y las altas temperaturas. No obstante, también se podría pensar que los métodos de control no fueron adecuados: zonas encharcadas sin drenar en las fincas con acceso a los animales, escasa aplicación de molusquicidas y fasciolicidas; o bien la existencia de resistencias de los mismos.

\section{CONCLUSIÓN}

La prevalencia $(5.61 \pm 2.82)$ de lesiones compatibles con fasciolosis, sigue estando presente en ganado vacuno. Los años en los que se encontraron mayor número de lesiones fueron 2014 y 2016 . Es pertinente una inspección exhaustiva por parte del veterinario de las vísceras del ganado de lidia postmortem en el desolladero, para valorar la eficacia de los tratamientos antiparasitarios y a la vez estudiar el posible efecto negativo de este parásito en el rendimiento físico del toro en el ruedo.

\section{AGRADECIMIENTOS}

Gracias a todos los ganaderos, compañeros veterinarios y empresarios taurinos por las facilidades en la toma de datos durante todos estos años.

\section{LITERATURA CITADA}

ALBUQUERQUE E, de Oliveira T, Lopes S, Menezes-Souza D, Castanheira S, Vilhena I, Maria L, dos Santos W. 2013. Expression of IL-4, IL-10 and IFN-in the liver tissue of cattle that are naturally infected with Fasciola hepatica. Veterinary Parasitology. 195:177- 182. https://doi.org/10.1016/j.vetpar.2013.03.035

ASTIZ-BLANCO S, Elvira Partida L, González Martín JV. 2007. Parasitología práctica en clínica de campo de vacuno. Redvet. 8(4):1-20. https://www.redalyc.org/articulo.oa?id=63613303007 
BEESLEY NJ, Williams DJ, Paterson S, Hodgkinson J. 2017. Fasciola hepatica demonstrates high levels of genetic diversity, a lack of population structure and high gene flow: possible implications for drug resistance. Int $J$ Parasitol. 47(1):11-20. https://doi.org/10.1016/j.ijpara.2016.09.007

CLARIDGE J, Diggle P, McCann CM, Mulcahy G, Flynn R, McNair J, Strain S, Welsh M, Baylis M, Williams D. 2012. Fasciola hepatica is associated with the failure to detect bovine tuberculosis in dairy cattle. Nature Communications. 3:853. https://doi.org/10.1038/ncomms1840

CLERY D, Torgerson P, Mulcahy G. 1996. Immune responses of chronically infected adult cattle to Fasciola hepatica. Veterinary Parasitology. 62:71-82. https://doi.org/ 10.1016/0304-4017(95)00858-6

CORDERO del Campillo M. 1990. Fasciolosis: revisión de algunos aspectos. Información Veterinaria. 87:32-40.

https://buleria.unileon.es/bitstream/handle/10612/3569/Informacion\%20Veterinaria\% 28Fasciolosis\%29\%28N\%c2\%ba\%2087\%29.pdf?sequence=1\&isAllowed=y

CUNNINGHAM J. 2003. Fisiología Veterinaria. Elsevier, Madrid. Pp. 575. ISBN: 9788481746594

DA SILVA A, Baldiserra M, Bottari N, Gabriel M, Rhoden L, Piva M, Christ R, Stedille F, Gris A, Morsch V, Schetinger MR, Mendes R. 2017. Oxidative stress and changes in adenosine deaminase activity of cattle experimentally infected by Fasciola hepatica. Parasitology. 144:520-526. https://doi.org/10.1017/S0031182016002043

DIEZ Baños MA, Rojo-Vázquez FA. 1976. Influencia de la temperatura en el desarrollo de los huevos de Fasciola hepática. Anal. Facultad. Vet. León. 22(1): 65-75. https://dialnet.unirioja.es/servlet/articulo?codigo=1961713

ESCALERA-VALENTE F, González-Montaña JR, Alonso de la Varga M, LomillosPérez JM, Gaudioso-Lacasa VR. 2013. Influence of intense exercise on acid-base, blood gas and electrolyte status in Bulls. Research in Veterinary Science. 95:623-628. https://doi.org/10.1016/j.rvsc.2013.03.018

ESCRIBANO B, Tunez I, Requena F, Rubio, M, De Miguel R, Montilla P, Tovar P, Aguera E, 2010. Effects of an aerobic training program on oxidative stress biomarkers in bulls. Veterinarni Medicina. 55:422-428. https://doi.org/10.17221/2979-VETMED

FORBES AB, Reddick D, Stear MJ. 2015. Efficacy of treatment of cattle for liver fluke at housing: influence of differences in flukicidal activity against juvenile Fasciola hepatica. Veterinary Record. 176(13):333. https://doi.org/10.1136/vr.102720. https://veterinaryrecord.bmj.com/content/176/13/333 
GARCÍA-RODRÍGUEZ JA, Martin Sánchez AM, Fernández Gorostarzu JM, García Luis EJ. 1985. Fascioliasis in Spain: A review of the literature and personal observations. Eur J Epidemiol. 1: 121-126. https://doi.org/10.1007/BF00141804

GARCÍA-SACRISTÁN A, Castejón Montijano F, de la Cruz Palomino LF, González Gallego J, López Murillo MD, Salido, Ruiz G. 1996. Fisiología Veterinaria. McGrawHill-Interamericana. Madrid, España. Pp. 1074. ISBN: 84-486·0126·2. Depósito legal: M. 25.204.1996

GRAIG TM, JHuey RL. 1984. Efficacy of tricabendazole against Fasciola hepatica y Fasciola Magna in naturally infected calves. J. Vet. Res. 46:1644-1647. PMID: 6476577.

HINEY K, Nielsen B, Rosenstein D, Orth M, Marks B. 2004. High-intensity exercise of short duration alters bovine bone density and shape. Journal of Animal Science. 82: 1612-1620. https://doi.org/10.2527/2004.8261612x

KANEKO JJ, Harvey JW, Bruss ML. 1997. Clinical Biochemistry of Domestic Animals. Academic Press, San Diego, California. USA. Pp. 932. ISBN: 9780080529196.

KELLEY JM, Elliott TP, Beddoe T, Anderson G, Skuce P, Spithill TW. 2016. Current Threat of Triclabendazole Resistance in Fasciola hepatica. Trends Parasitol. 32(6):458-469. https://doi.org/10.1016/j.pt.2016.03.002

LOMILLOS JM, Alonso ME, Sánchez-García C, Gaudioso VR. 2012. Evolución del sector de la producción del toro de lidia en España. Censos y ganaderías. Revista ITEA. 108(2):207-221. http://www.aida-itea.org/aida-itea/files/itea/revistas/2012/1082/(207-221)\%20A2288\%20ITEA\%20108-2.pdf

LOMILLOS JM, Alonso ME. 2018. Evolución del síndrome de caída del toro de lidia en los últimos 25 años. Abanico Veterinario. 8(1):80-90. http://dx.doi.org/10.21929/abavet2018.81.8

MULONE JB 1986. Fascioliasis and cestodiasis in cattle. Veterinary Clinics of North America. Food Animal Practice. 2(2):261-75. https://doi.org/10.1016/S07490720(15)31236-6

MARCOS LA, Yi P, Machicado A, Andrade R, Samalvides F, Sanchez J and Terashima A. 2007. Hepatic fibrosis and Fasciola hepatica infection in cattle. Journal of Helminthology. 81:381-386. https://doi.org/10.1017/S0022149X07850231

MOONEY L, Good B, Hamraham JP, Mulcahy G, De Wool T. 2009. The comparative efficacy of four antihelmintics against a natural acquired Fasciola hepatica infection in hill sheep flock in the west of Ireland. Vet. Veterinary Parasitology. 164(2-4):201-205. https://doi.org/10.1016/j.vetpar.2009.05.017 
NOGAREDA C, Mezo M, Uriarte J, Lloveras J, Cordero del Campillo M. 2006. Dynamics of infestation of cattle and pasture by gastrointestinal nematodes in an Atlantic temperate environment. Journal of Veterinary Medicine. 53(9):439-44. https://doi.org/10.1111/j.1439-0450.2006.00979.x

ORJALES I, Mezo M, Miranda M, González-Wartella M, Rey-Crespo F, Vaarst M, Thamsborg S, Diéguez FJ, Castro-Hermida JA, López-Alonso M. 2017. Helminth infections on organic dairy farms in Spain. Veterinary Parasitology. 243:115-118. https://doi.org/10.1016/j.vetpar.2017.06.031

PAINCEIRA AM. 2012. Prevalencia y factores de riesgo asociados a la infección por endoparásitos en rumiantes domésticos y silvestres de la provincia de Lugo. Tesis Doctoral. Universidad Santiago de Compostela. España. https://minerva.usc.es/xmlui/bitstream/handle/10347/6124/rep_262.pdf?sequence=1 \&isAllowed=y

PÉREZ R, Recabarren S, Islas A, Jara C, Valdés P, Hetz E. 1992. Glucosa, ácido láctico y equilibrio ácido-base en equinos de tiro sometidos a ejercicio de tracción prolongada. Archivos de Medicina Veterinaria. 23:43-51. http://mingaonline.uach.cl/scielo.php?script=sci_arttext\&pid=S0301732X1997000100 005\&lng=es\&nrm=iso

PIÑEIRO P. 2013. Estudio de los posibles reservorios de la fasciolosis en Galicia. Tesis Doctoral. Universidad Santiago de Compostela. España. http://hdl.handle.net/10347/9834

REAL DECRETO 60/2001, de 26 de enero, sobre prototipo racial de la raza bovina de lidia. BOE núm. 38 Martes 13 febrero 2001.

REAL DECRETO 260/2002, de 8 de marzo, por el que se fijan las condiciones sanitarias aplicables a la producción y comercialización de carnes de reses de lidia.

ROJO-VÁZQUEZ FA, Meana A, Valcárcel F, Martínez-Valladares M. 2012. Update on trematode infections in sheep. Vet Parasitol. 189(1):15-38. https://doi.org/ 10.1016/j.vetpar.2012.03.029

ROWCLIFFE SA, Ollerenshaw CB. 1960. Observations on the bionomics of the egg of Fasciola hepatica. Ann Trop Med Parasitol. 54:172-81. https://doi.org/ 10.1080/00034983.1960.11685973

Publica en las revistas Abanico. Publish in Journals Abanico. 